\title{
Hydroxypropylcellulose as a novel green reservoir for the synthesis, stabilization, and storage of silver nanoparticles
}

This article was published in the following Dove Press journal:

International Journal of Nanomedicine

16 March 2015

Number of times this article has been viewed

\author{
Muhammad Ajaz Hussain' \\ Abdullah Shah' \\ Ibrahim Jantan² \\ Muhammad Raza Shah ${ }^{3}$ \\ Muhammad Nawaz Tahir ${ }^{4}$ \\ Riaz Ahmad $^{5}$ \\ Syed Nasir Abbas Bukhari ${ }^{2}$ \\ 'Department of Chemistry, University \\ of Sargodha, Sargodha, Pakistan; \\ ${ }^{2}$ Drug and Herbal Research Centre, \\ Faculty of Pharmacy, Universiti \\ Kebangsaan Malaysia, Jalan Raja Muda \\ Abdul Aziz, Kuala Lumpur, Malaysia; \\ ${ }^{3}$ International Center for Chemical \\ and Biological Sciences, University \\ of Karachi, Karachi, Pakistan; ${ }^{4}$ Institute \\ of Inorganic and Analytical Chemistry, \\ Johannes Guttenberg University, \\ Duesbergweg, Mainz, Germany; \\ ${ }^{5}$ Centre for Advanced Studies \\ in Physics (CASP), GC University, \\ Lahore, Pakistan
}

\begin{abstract}
Polysaccharides are attracting the vigil eye of researchers in order to design the green synthesis of silver nanoparticles (Ag NPs) of diverse size, shape, and application. We report an environmentally friendly method to synthesize Ag NPs where no physical reaction conditions were employed. Hydroxypropylcellulose (HPC) was used as a template nanoreactor, stabilizer, and capping agent to obtain $\mathrm{Ag}$ NPs. Different concentrations of $\mathrm{AgNO}_{3}$ solutions (50 mmol, $75 \mathrm{mmol}$, and $100 \mathrm{mmol}$ ) were mixed with a concentrated aqueous solution of HPC and the progress of the reaction was monitored by noting color changes of the reaction mixture at different reaction times for up to 24 hours. Characteristic ultraviolet-visible spectroscopy (UV/Vis) absorption bands of Ag NPs were observed in the range of 388-452 nm. The morphology of the Ag NPs was studied by scanning electron microscopy, transmission electron microscopy (TEM), and atomic force microscopy. The TEM images confirmed that the size of the Ag NPs was in the range of 25-55 nm. Powder X-ray diffraction studies showed that the crystal phase of the Ag NPs was face-centered cubic. The as-prepared Ag NPs were found to be stable, and no changes in size and morphology were observed after storage in HPC thin films over 1 year, as indicated by UV/Vis spectra. So, the present work furnishes a green and economical strategy for the synthesis and storage of stable Ag NPs. As-synthesized Ag NPs showed significant antimicrobial activity against different bacterial (Escherichia coli, Staphylococcus epidermidis, S. aureus, Bacillus subtilis, Pseudomonas aeruginosa) and fungal strains (Actinomycetes and Aspergillus niger).
\end{abstract}

Keywords: green synthesis, nanoreactor, nanobiotechnology, antimicrobial assay, storage, stability

\section{Introduction}

Metal nanoparticles (NPs) have versatile applications due to their unique electronic, physicochemical, and optical properties as compared to their bulk counterpart. ${ }^{1-3}$ Among metal NPs, silver (Ag) NPs hold a place of distinction due to many desirable attributes such as their catalytic activity, electrical conductivity, ${ }^{4,5}$ anticancer ${ }^{6,7}$ and antibacterial properties, ${ }^{8,9}$ as well as their applications in bioimaging ${ }^{10}$ and biosensors. ${ }^{11}$ The synthesis of metal NPs has been achieved through different methods, such as electrochemical engineering, ${ }^{12,13}$ irradiation, ${ }^{14,15}$ and chemical reduction of $\mathrm{Ag}$ salts in solution. ${ }^{16,17}$

The synthesis of Ag NPs by the chemical reduction method involves toxic solvents, ${ }^{18}$ dangerous reducing agents like sodium borohydride, ${ }^{19,20}$ and synthetic/nonbiodegradable stabilizing agents. ${ }^{21,22}$ Such synthesis strategies may also result in biological hazards and environmental toxicity. Secondly, Ag NPs have a high surface area-to-volume ratio, resulting in their aggregation with the passage of time that limits their potential 
applications. Therefore, there has been a need to develop synthesis protocols involving nontoxic, biodegradable, and low-cost green chemicals ${ }^{23,24}$ for the synthesis of Ag NPs. ${ }^{25,26}$

Polysaccharides are getting attention as an in situ reducing and self-capping agent for the green synthesis of Ag NPs. ${ }^{27-30}$ Additionally, the film-forming properties of polysaccharides can be exploited for the long-term storage of Ag NPs without agglomeration. Recently, cellulose ether derivatives have been used for the synthesis of Ag NPs. It was recently reported that hydroxyethylcellulose could be used for the green synthesis of Ag NPs. However, the reactions require elevated temperatures around $70^{\circ} \mathrm{C}$ and the use of strong basic reaction conditions ( $\mathrm{pH} 12) .{ }^{27}$ Therefore, a need is felt to introduce new cellulose ether derivatives, which may perform reactions under mild conditions. Hence, we aimed to exploit another cellulose ether derivative, ie, hydroxypropylcellulose (HPC), as a reducing agent, in situ stabilizer, and capping agent for the synthesis of Ag NPs. The proposed green and controlled synthesis of stable Ag NPs is achieved without any physical reaction conditions (like temperature or $\mathrm{pH}$ ) by simply exposing the concentrated aqueous solution of HPC and $\mathrm{AgNO}_{3}$ to sunlight. We also demonstrate the use of HPC thin films as a storage medium for as-synthesized Ag NPs.

\section{Experimental methods Materials}

$\mathrm{AgNO}_{3}$ (99.98\%) was purchased from EMD Millipore (Billerica, MA, USA). HPC (MS 3.46, 60\% HP moieties) was obtained from Nanjing Yeshun Industry \& International Trading Co., Ltd (Jiangsu, People's Republic of China). Other reagents and analytical grade solvents were obtained from Fluka (Sigma-Aldrich Co., St Louis, MO, USA). All aqueous solutions were prepared in deionized water.

\section{Green synthesis of Ag NPs mediated by HPC}

A concentrated solution of HPC was prepared in deionized water. The $\mathrm{AgNO}_{3}$ solution (50 mmol) was then mixed with the HPC solution in equal volume at room temperature and kept under dark conditions. The mixture was subsequently exposed to sunlight and the progress of the reaction was monitored by ultraviolet-visible spectroscopy (UV/Vis) analysis. The same procedure was opted for different concentrations of $\mathrm{AgNO}_{3}(75 \mathrm{mmol}$ and $100 \mathrm{mmol})$ solutions, respectively.

\section{Film formation of HPC loaded with} Ag NPs

The formation of Ag NPs embedded in HPC thin films was carried out by drying a mixture of $\mathrm{HPC}$ and $\mathrm{AgNO}_{3}$
( $50 \mathrm{mmol}, 75 \mathrm{mmol}$, and $100 \mathrm{mmol}$ ) after being exposed to diffused sunlight for 12 hours. The reaction mixture was then dried to thin films under air and dark conditions in a Petri dish. The resultant thin dry films were characterized using UV/Vis spectrophotometry, scanning electron microscopy (SEM), transmission electron microscopy (TEM), and power $\mathrm{X}$-ray diffraction (PXRD).

\section{UV/Vis spectrophotometry}

The progress of the reduction of $\mathrm{Ag}^{+}$under diffused sunlight (ultraviolet [UV] irradiation) was monitored by recording the $\mathrm{UV} / \mathrm{Vis}$ spectra of the reaction mixtures in a wavelength range of $800-200 \mathrm{~cm}^{-1}$ on the UV/Vis spectrophotometer, UV-1700 PharmaSpec (Shimadzu Corporation, Kyoto, Japan).

\section{FT-IR spectroscopy}

Fourier transform infrared (FT-IR) spectra of pure HPC and HPC loaded with Ag NPs were recorded on an IR Prestige-21 (Shimadzu Corporation) spectrometer using the KBr pellet technique. The pellets were dried under vacuum before analysis.

\section{SEM}

The size and morphology of the Ag NPs were observed using SEM. Microtomes were sliced from HPC thin films loaded with Ag NPs and analyzed on SEM with carbon stubs (carbon adhesive Leit-Tabs No. G 3347) (PLANO GmbH, Wetzlar, Germany).

\section{TEM}

Freshly prepared Ag NP solutions were centrifuged and the isolated Ag NPs were studied by TEM on a Philips 420 instrument with an acceleration voltage of $120 \mathrm{kV}$. The 1 -year stored Ag NP-embedded HPC thin films were dissolved in deionized water and the Ag NPs were isolated by centrifugation before the TEM measurements. The samples were prepared by drop casting on a carbon-coated $\mathrm{Cu}$ TEM grid.

\section{AFM}

An HPC thin film loaded with Ag NPs was dissolved in deionized water. The sample was dropped on a freshly cleaved mica sheet and dried overnight. The morphology of the Ag NPs was observed using an AFM, Multimode, Nanoscope IIIa (Veeco Instruments, Inc., Plainview, NY, USA) in tapping mode.

\section{PXRD}

The crystal structure of the Ag NPs was determined on an X'Pert PRO MPD (PANalytical, Almelo, the Netherlands) 
diffractometer (over a range of $5^{\circ}-100^{\circ}, 2 \theta$ ), equipped with monochromatic X-rays.

\section{Antimicrobial activity of Ag NPs}

The bacterial strains Escherichia coli (American Type Culture Collection [ATCC] 25922), Bacillus subtilis (ATCC 6633), Staphylococcus aureus (ATCC 25923), Pseudomonas aeruginosa (ATCC 27853), and S. epidermidis (ATCC 12228), as well as fungal strains (Actinomycetes and Aspergillus niger) were used to carry out the antimicrobial assay of pristine Ag NPs. Bacterial strains were grown over Mueller-Hinton agar media (Oxoid Ltd., Hampshire, UK), while fungal growth was performed over Sabouraud dextrose agar (Hardy Diagnostics, Santa Maria, CA, USA). Inoculums (microbial culture in their respective media, $10 \mathrm{~mL}$ ) were inoculated for 24 hours at $37^{\circ} \mathrm{C}$ for bacteria and for $30-37$ hours at $27^{\circ} \mathrm{C}-30^{\circ} \mathrm{C}$ for fungal strains. Fungal strain culture (7 days old) was washed and suspended in normal saline solution, filtered through aseptic glass wool, and incubated at $28^{\circ} \mathrm{C}$. To accelerate the growth of the microbial strains, the tubes were shaken periodically. The turbidity of the inoculums was adjusted by $0.5 \mathrm{McF}$ arland Standards.

The antimicrobial assay of Ag NPs was carried out by the widely used disc (6 mm discs of Whatman number 1 filter paper) diffusion method. Mueller-Hinton agar plates for bacterial strains were used, upon which the inoculum $(0.1 \mathrm{~mL})$ was spread uniformly. Ag NP-loaded discs were carefully placed on the surface of the culture medium. The culture plates were incubated for 24 hours at $37^{\circ} \mathrm{C}$. A pure dimethyl sulfoxide-loaded (15-20 mL) disc was used as the negative control. All of the assays were performed in triplicate and the mean values were reported.

\section{Results and discussion Synthesis of Ag NPs}

The preparation of Ag NPs by the UV irradiation method produces hydrated electrons in the reaction system, which can be used to reduce Ag ions into zero-valent metallic Ag NPs. Hence, the use of additional toxic and environmentally dangerous reducing agents can be circumvented. ${ }^{19,27}$ Here, we have synthesized Ag NPs under diffused sunlight using $\mathrm{HPC}$ as an in situ capping and reducing agent.

$\mathrm{AgNO}_{3}$ solution containing the $\mathrm{Ag}^{+}$was mixed with HPC solution. The Ag ion reacted with HPC to produce an $(\mathrm{Ag}[\mathrm{HPC}])^{+}$complex. The $(\mathrm{Ag}[\mathrm{HPC}])^{+}$complex was subsequently exposed to sunlight radiation. $\mathrm{The}^{\mathrm{Ag}^{+}}$was reduced by $\mathrm{HPC}$ polymer to $(\mathrm{Ag}[\mathrm{HPC}])$ as an intermediate precursor. The negative charge on the hydroxyl groups in the HPC backbone capped the positively-charged groups on the surface of the Ag NPs resulting in the colloidal stabilization of (Ag[HPC]).

The formation of Ag NPs upon irradiation of the $(\mathrm{Ag}[\mathrm{HPC}])^{+}$intermediate complex was indicated by a change in the color of the reaction mixture. Solutions changed from colorless to reddish-brown with progress of the reaction observed over a period of 6 hours. The photographs showing the color change on exposure to diffused sunlight are shown in Figure 1.

\section{Characterization of Ag NPs UV/Vis spectrophotometry}

$\mathrm{AgNO}_{3}$ solutions $(50 \mathrm{mmol}, 75 \mathrm{mmol}$, and $100 \mathrm{mmol} ; 2 \mathrm{~mL}$ aliquot each) were mixed with concentrated HPC solution $(2 \mathrm{~mL})$ and the progress of the reaction was monitored by
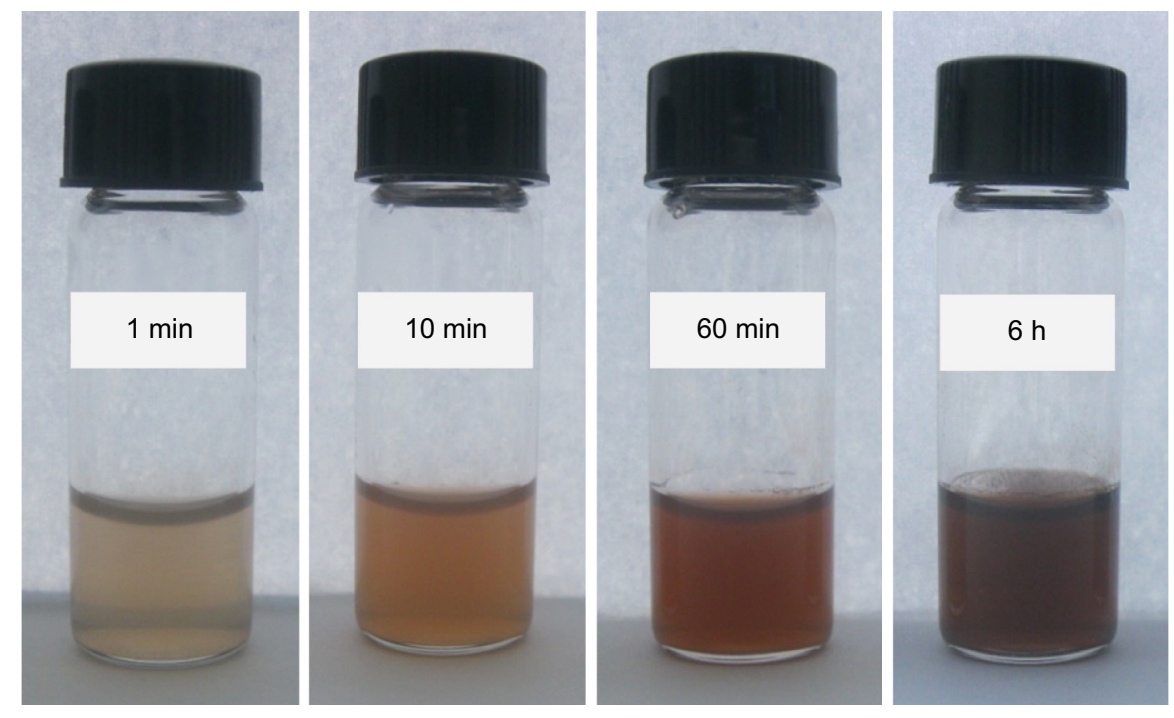

Figure I Photographs of $\mathrm{HPC}-\mathrm{Ag}^{+}$solutions $\left(50 \mathrm{mmol} \mathrm{AgNO}_{3}\right)$ showing color change with a reduction of $\mathrm{Ag}^{+}$upon exposure to diffused sunlight. Abbreviations: min, minutes; h, hours; HPC, hydroxypropylcellulose. 
$\mathrm{UV} / \mathrm{V}$ is spectrophotometry. $\mathrm{Ag}^{+}$was reduced to $\mathrm{Ag}$ NPs upon exposure to sunlight. The collective oscillation of conduction electrons in Ag NPs results in the surface plasmon resonance (SPR) phenomenon. This SPR causes a strong absorption by Ag NPs in the visible region. ${ }^{31}$ Depending upon the particle size and reaction time, the SPR transitions of Ag NPs showed color changes in the reaction mixture from yellowish-brown to reddish-brown. UV/Vis absorption bands were observed at $388 \mathrm{~nm}, 418 \mathrm{~nm}, 432 \mathrm{~nm}, 438 \mathrm{~nm}$, and $448 \mathrm{~nm}$ for $50 \mathrm{mmol} ; 390 \mathrm{~nm}, 419 \mathrm{~nm}, 437 \mathrm{~nm}, 445 \mathrm{~nm}$, and $451 \mathrm{~nm}$ for $75 \mathrm{mmol}$; and $416 \mathrm{~nm}, 432 \mathrm{~nm}, 440 \mathrm{~nm}$,

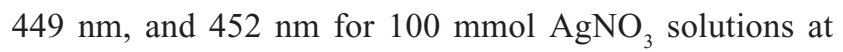
0.5 hours, 1 hour, 2 hours, 5 hours, and 24 hours, respectively. The results are in good agreement with the Ag NPs produced by another cellulose ether (ie, hydroxyethylcellulose), where exogenous reducing agents were employed. ${ }^{27}$ The absorption peaks showed a red shift, which means that the size of the Ag NPs increased with an increase in reaction time. Likewise, an increase in absorption intensity was also noticed with an increase in reaction time from 30 minutes to 24 hours, indicating the continuous reduction of $\mathrm{Ag}^{+}$by HPC with the passage of time. The results of the UV/Vis analyses are summarized in Figure 2.

A

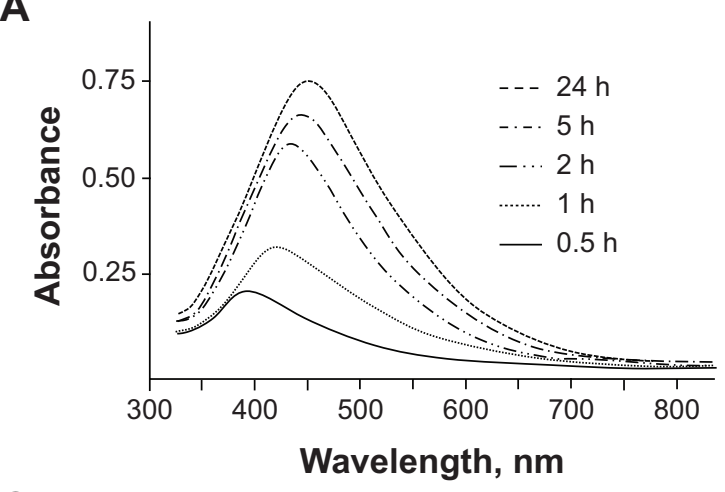

C

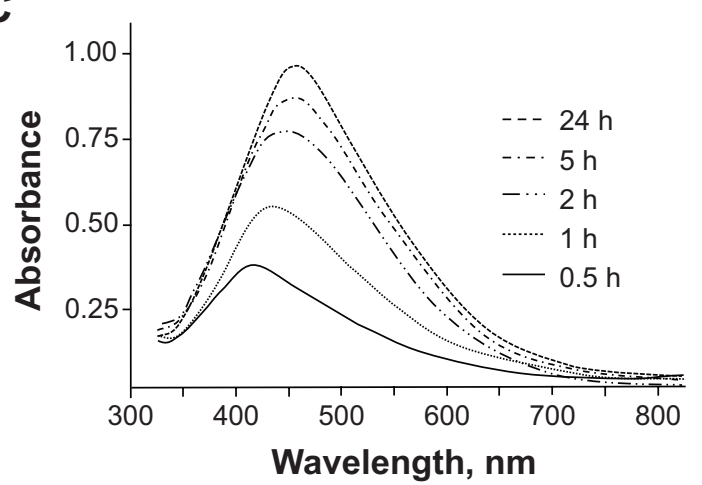

\section{FT-IR spectroscopy}

The interactions between hydroxyl groups of HPC and Ag NPs were studied by FT-IR spectroscopy (Figure 3). The peak at $1,051 \mathrm{~cm}^{-1}$ due to C-O-C stretching of HPC was changed to a broad band in the range of 1,000-1,113 $\mathrm{cm}^{-1}$ for HPC-Ag NPs. Ag---O van der Waal interactions showed a band in the range of 472-594 $\mathrm{cm}^{-1}$, which is not observed in the pure HPC spectrum. All other bands were observed at positions that were quite similar to pure HPC. The band in the range of 594-646 cm $\mathrm{cm}^{-1}$ in HPC was also broadened after the incorporation of Ag NPs. Similar FT-IR spectra were observed for $50 \mathrm{mmol}$ and $100 \mathrm{mmol}$ solutions as well.

\section{SEM of films}

Microtomes sliced from the HPC thin films embedded with Ag NPs were analyzed by SEM to see the distribution of Ag NP-embedded thin films. The uniform dispersion of Ag NPs in HPC thin films was therefore witnessed by SEM images. The results are shown in Figure 4.

\section{TEM of isolated Ag NPs}

HPC thin films embedded with Ag NPs were dissolved in distilled water, and isolated Ag NPs were separated using

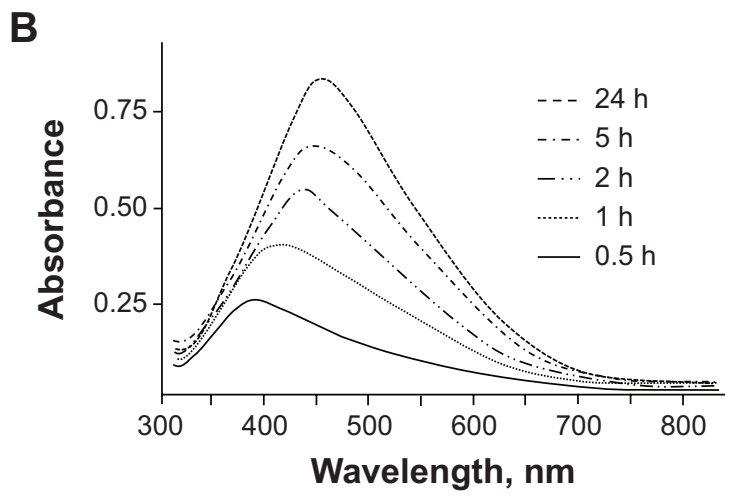

D

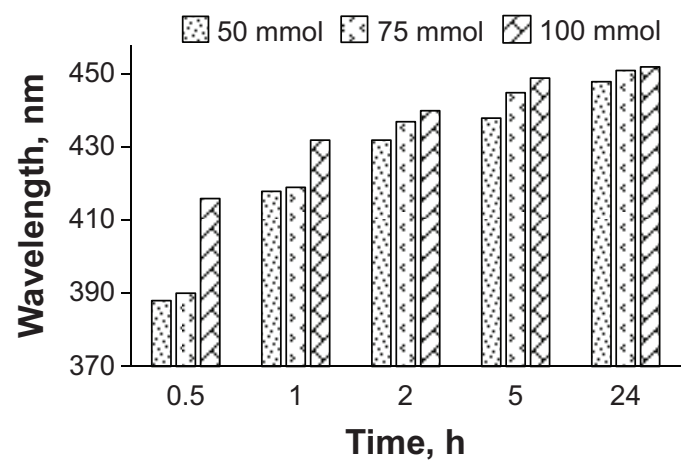

Figure $2 \mathrm{UV} / \mathrm{Vis}$ spectra of $\mathrm{Ag} \mathrm{NPs}$ prepared using HPC.

Notes: (A) $50 \mathrm{mmol}$, (B) $75 \mathrm{mmol}$, and (C) $100 \mathrm{mmol}$ solution of $\mathrm{AgNO}_{3}$ at different reaction times, as well as their (D) cumulative graphical representation showing increase in absorption of the $\mathrm{Ag}$ NP solutions with an increase in reaction time and concentration.

Abbreviations: h, hours; UV/Vis, ultraviolet-visible spectroscopy; NPs, nanoparticles; HPC, hydroxypropylcellulose. 


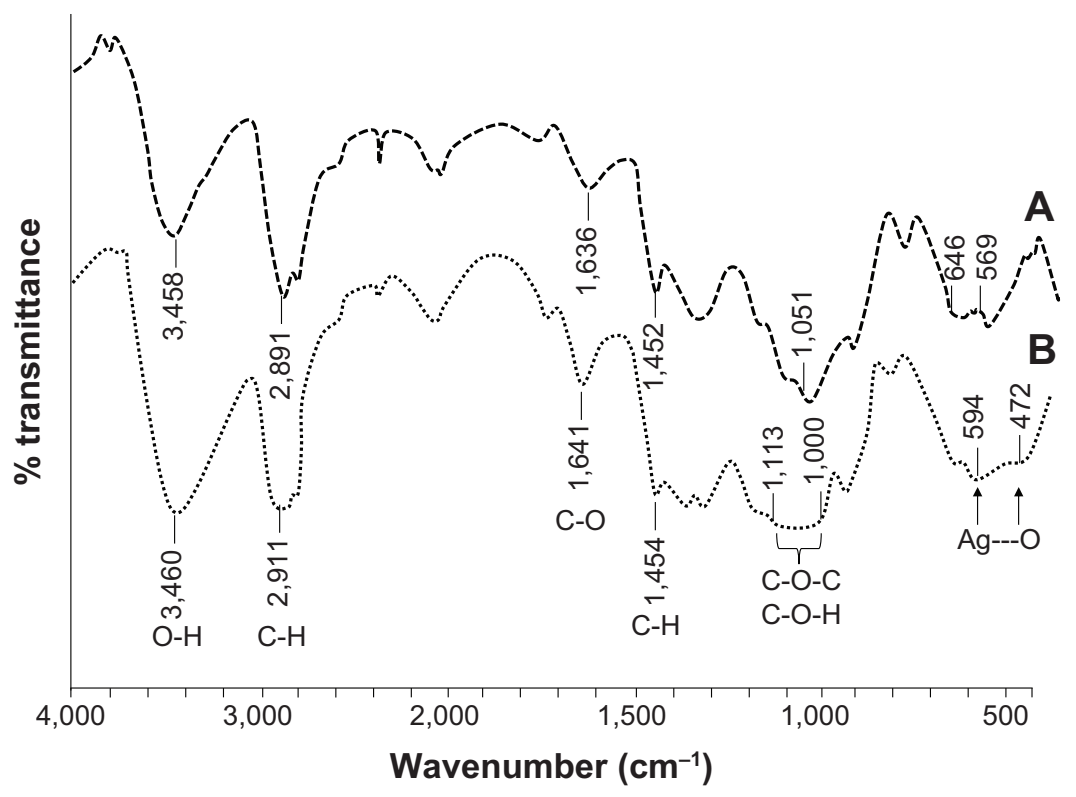

Figure $3 \mathrm{FT}-\mathrm{IR}(\mathrm{KBr})$ spectra of pure HPC and HPC films loaded with $\mathrm{Ag}$ NPs prepared from a 75 mmol $\mathrm{AgNO}_{3}$ solution.

Notes: (A) Pure HPC; (B) HPC films loaded with Ag NPs.

Abbreviations: FT-IR, Fourier transform infrared; HPC, hydroxypropylcellulose; NPs, nanoparticles.

centrifugation. The isolated Ag NPs were analyzed by TEM to assess the size distribution and morphology of the Ag NPs. The TEM images confirmed the formation of highly spherical Ag NPs with a size distribution in the range of $25-45 \mathrm{~nm}$, 25-50 nm, and 25-55 nm for $50 \mathrm{mmol}, 75 \mathrm{mmol}$, and 100 mmol $\mathrm{AgNO}_{3}$ solutions, respectively (Figure 5). TEM images of the Ag NPs isolated from the 1-year stored sample $(100 \mathrm{mmol})$ were also recorded. It was noted that Ag NPs retained their size and morphology after 1 year of storage, which further confirmed the useful storage properties of HPC thin films.

\section{PXRD}

The crystal phase of the as-synthesized Ag NPs isolated by centrifugation was confirmed by PXRD analyses of $50 \mathrm{mmol}$, $75 \mathrm{mmol}$, and $100 \mathrm{mmol}$ solutions (Figure 6) over the range of $5^{\circ}-100^{\circ}, 2 \theta$. The crystal phase of face-centered cubic Ag NPs was inferred from the diffraction peaks centered at (111), (200), (220), and (311) in all samples. The X-ray diffraction of the isolated Ag NPs after storing the Ag NPs in HPC thin films for 1 year was also recorded. The results revealed that there was also no change in the diffraction pattern of the stored sample. Therefore, it is inferred that
A

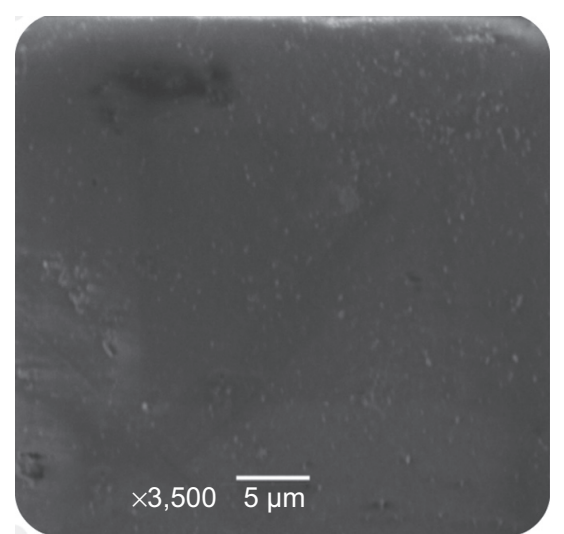

B

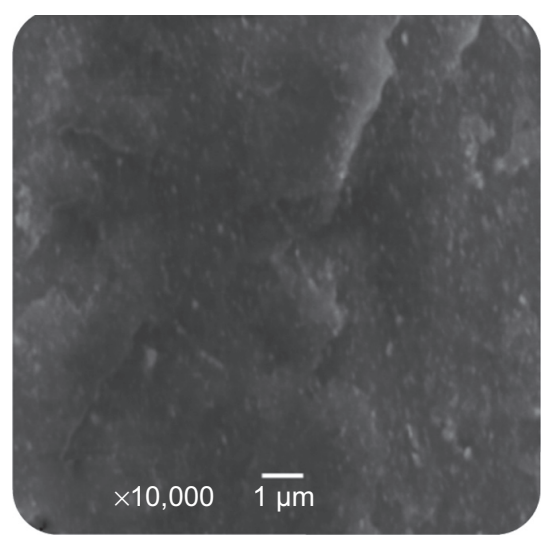

C

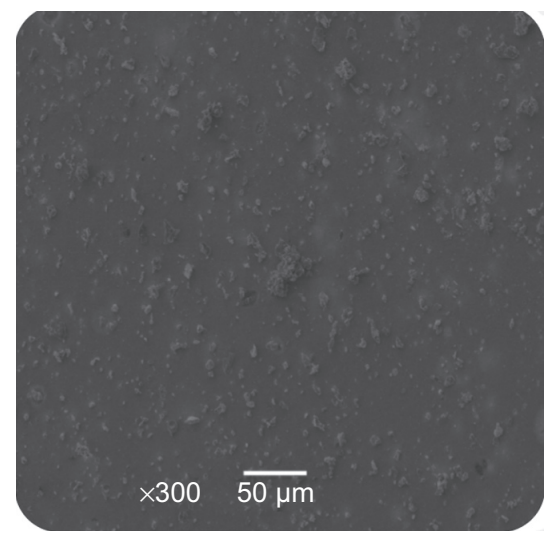

Figure 4 SEM images of Ag NPs embedded in HPC thin films.

Notes: (A) $50 \mathrm{mmol}$, (B) $75 \mathrm{mmol}$, and (C) $100 \mathrm{mmol} \mathrm{AgNO}_{3}$ solutions showing the distribution of $\mathrm{Ag} \mathrm{NPs}$.

Abbreviations: SEM, scanning electron microscopy; NPs, nanoparticles; HPC, hydroxypropylcellulose. 

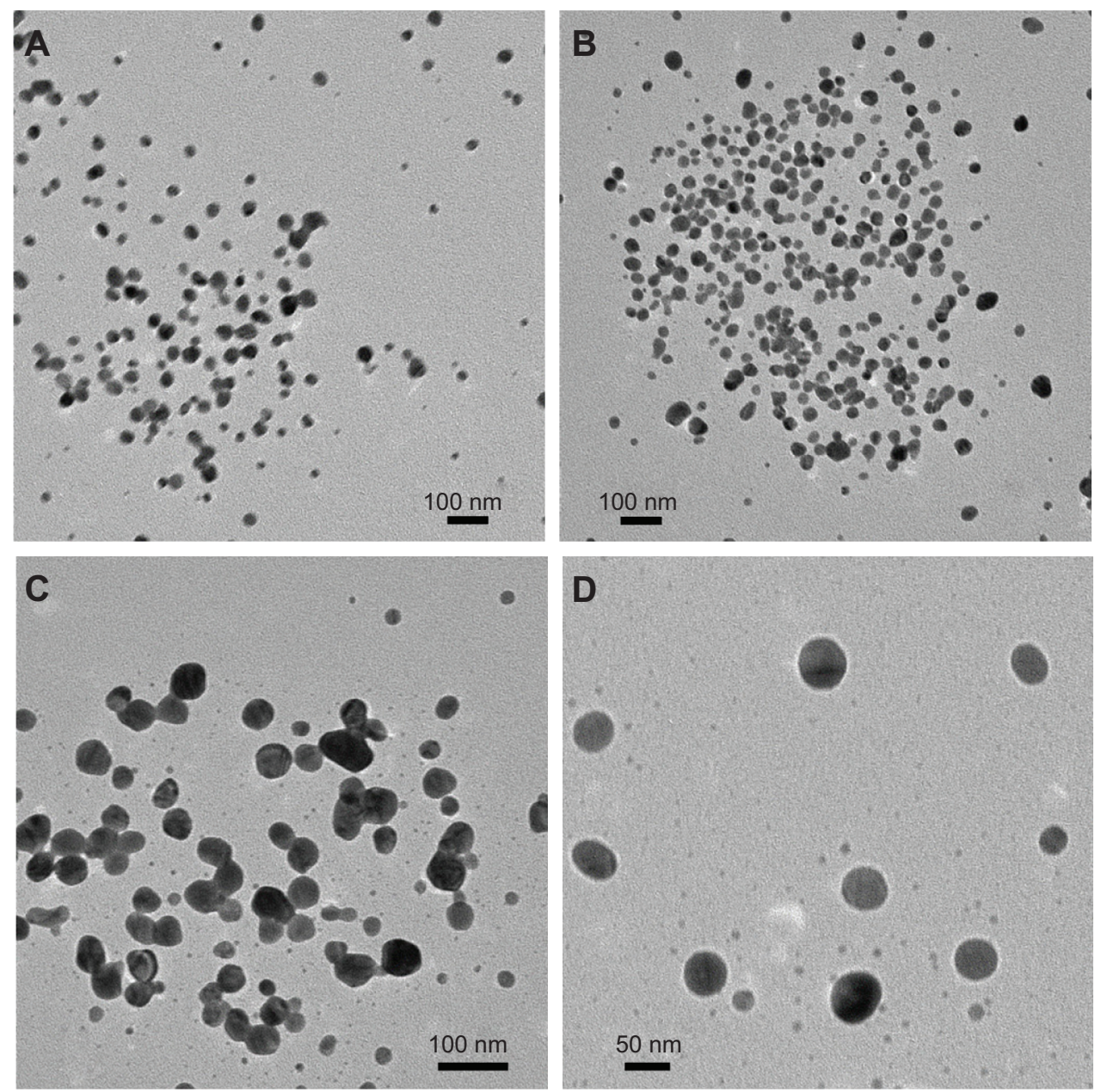

Figure 5 TEM images of isolated $\mathrm{Ag} \mathrm{NPs}$ from $50 \mathrm{mmol}, 75 \mathrm{mmol}, 100 \mathrm{mmol}$, and $100 \mathrm{mmol}$ ( 1 -year stored sample) $\mathrm{AgNO}_{3}$ solutions reduced by $\mathrm{HPC}$. Notes: (A) $50 \mathrm{mmol}$, (B) $75 \mathrm{mmol}$, (C) $100 \mathrm{mmol}$, and (D) $100 \mathrm{mmol}$ (I-year stored sample).

Abbreviations: TEM, transmission electron microscopy; NPs, nanoparticles; HPC, hydroxypropylcellulose.

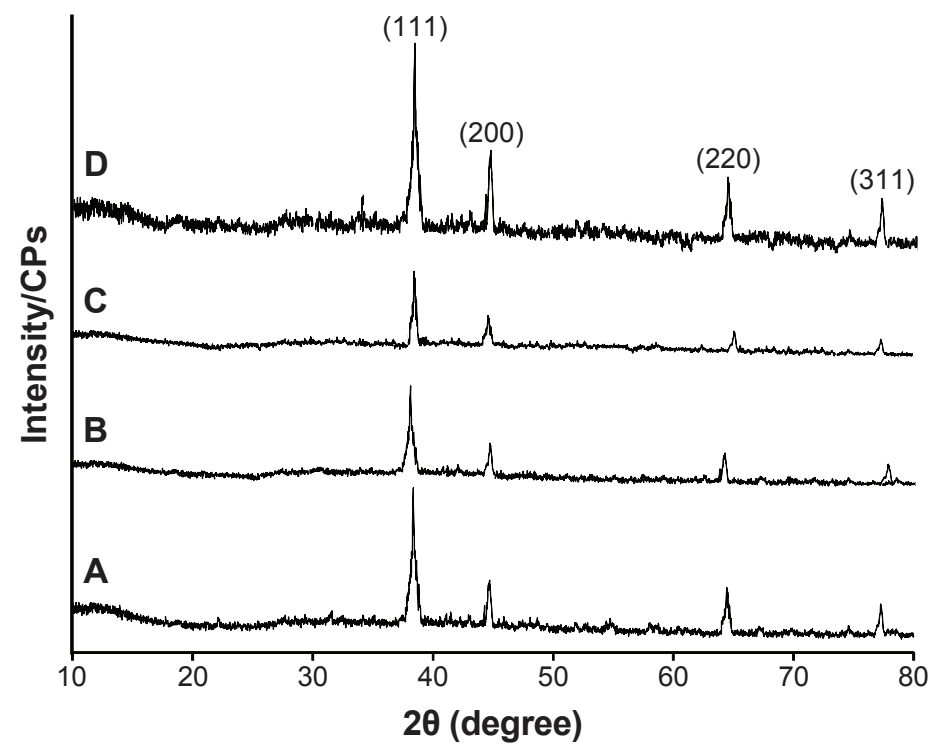

Figure 6 PXRD spectra of isolated Ag NPs.

Notes: (A) $50 \mathrm{mmol}$, (B) $75 \mathrm{mmol}$, and (C) $100 \mathrm{mmol}$ and (D) $50 \mathrm{mmol}$ (recorded after a l-year storage period).

Abbreviations: CPs, counts per second; PXRD, power X-ray diffraction; NPs, nanoparticles. 
the HPC thin films can be utilized for the long-term storage of Ag NPs.

\section{AFM}

Well-dispersed Ag NPs showed a spherical surface morphology in the AFM images (Figure 7). The capping of Ag NPs by HPC prevented their aggregation. Thus, it was concluded that HPC can be used as stabilizer and storage medium for Ag NPs.

\section{Storage of HPC-Ag NPs in thin films}

HPC was also evaluated as a storage medium for Ag NPs in solution, as well as in thin films. For this purpose, the $\mathrm{AgNO}_{3}$ solution $(100 \mathrm{mmol})$ was reduced by HPC for 12 hours under sunlight. The UV/Vis absorption spectra were recorded before and after 30 days' storage of the samples under dark, and comparable absorption maxima were obtained for the sample. A similar sample was dried and stored under dark conditions after UV/Vis irradiation for 12 hours. The resultant see-through thin films were stored for 1 year and UV analysis was recorded after dissolving the films in deionized water. No significant changes in the absorption intensity and shape of the UV/Vis spectra indicated that there was no sign of agglomeration of the Ag NPs during storage on the thin films. The SEM analyses of the 1-year stored Ag NP-embedded HPC thin films have further confirmed the hypothesis, and results symmetrical to the SEM images of the freshly prepared thin films (see Figure 4) were obtained. All of the storage results are depicted in Figure 8.
A

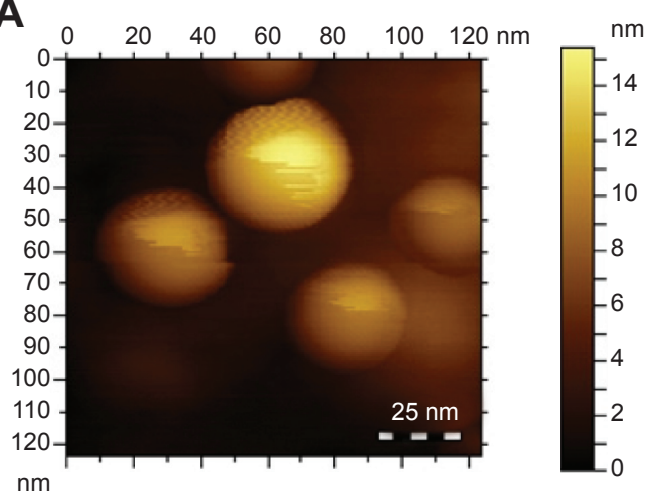

C

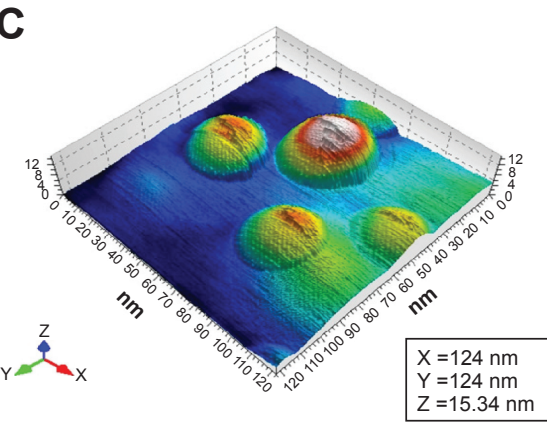

E
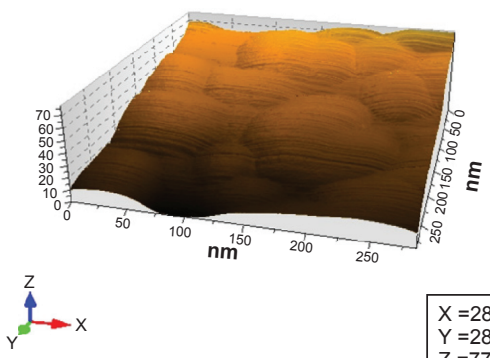

B
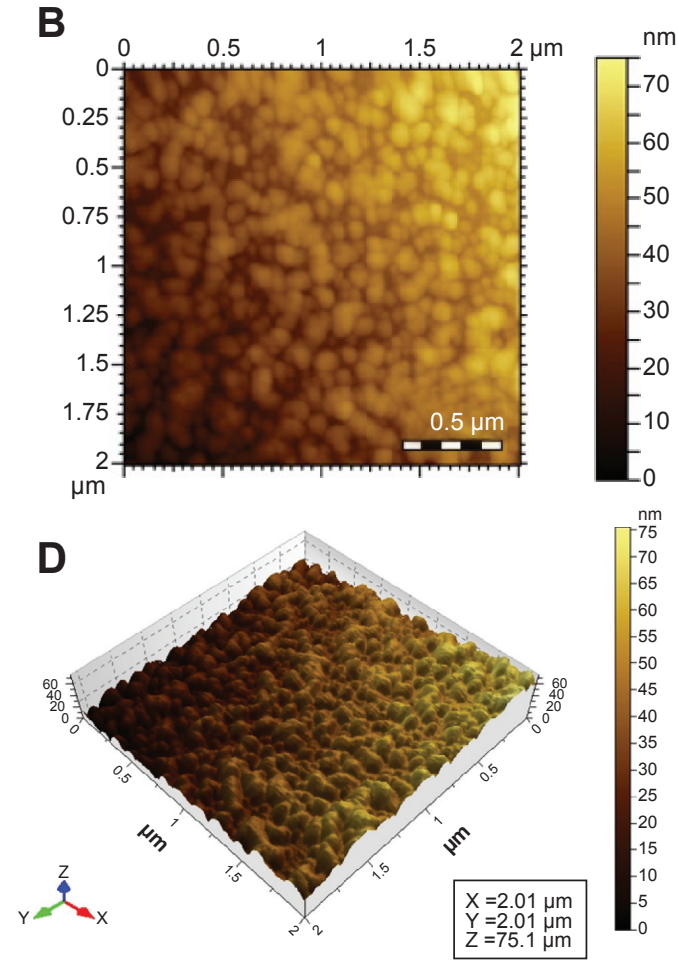

$\mathbf{F}$

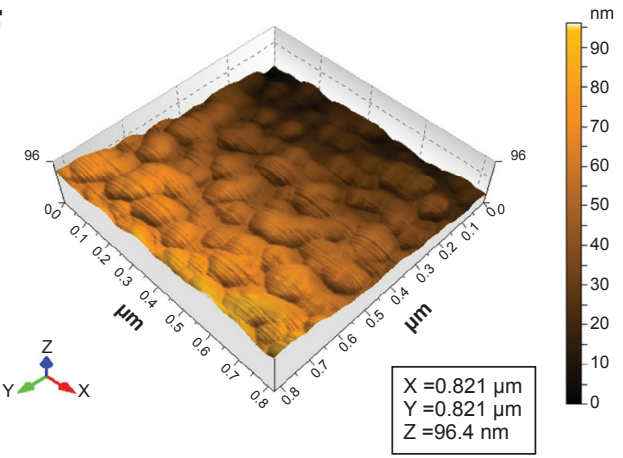

Figure 7 AFM images of Ag NPs embedded in HPC thin films confirming the symmetrical and spherical geometry of the Ag NPs.

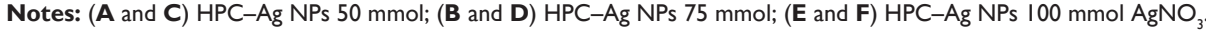

Abbreviations: AFM, atomic force microscopy; NPs, nanoparticles; HPC, hydroxypropylcellulose. 
A

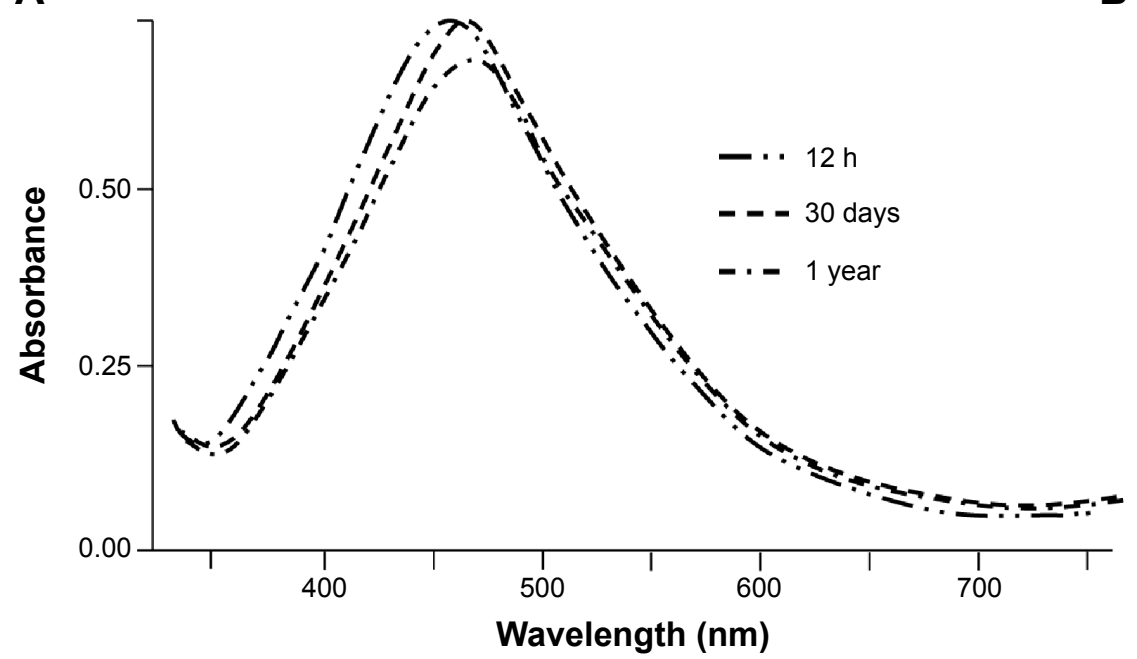

B

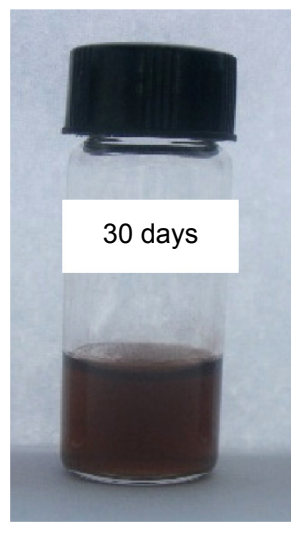

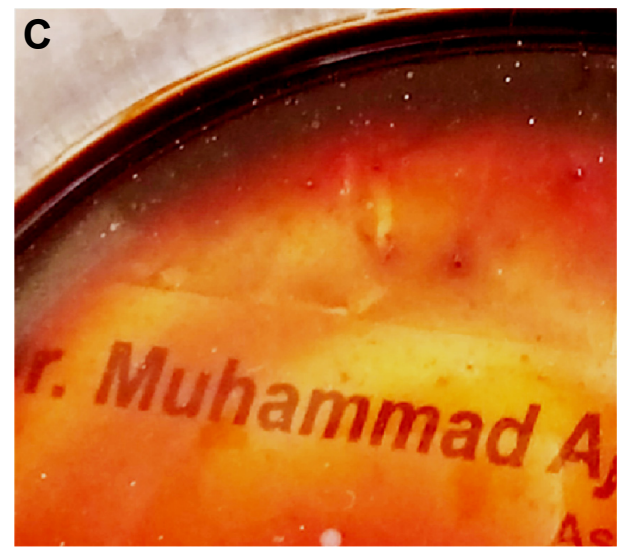

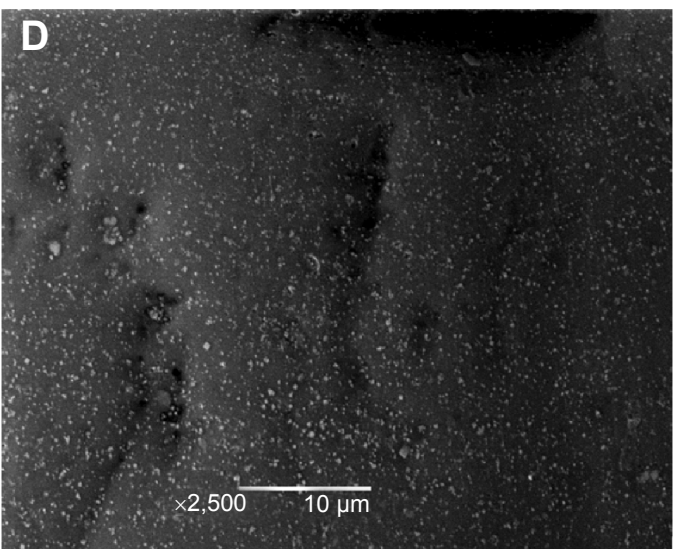

Figure $8 \mathrm{UV} / \mathrm{Vis}$ spectra of a $100 \mathrm{mmol} \mathrm{AgNO}_{3}$ solution in HPC, a vial indicating the color of the solution, see-through Ag NP-embedded HPC thin film, and SEM image of thin films taken after I year of storage under dark conditions.

Notes: (A) UV/Vis spectra of a $100 \mathrm{mmol} \mathrm{AgNO}_{3}$ solution in HPC recorded after $12 \mathrm{~h}, 30$ days, and I year of storage. (B) Vial indicating the color of the solution. (C) Seethrough Ag NP-embedded HPC thin film. (D) SEM image of thin films taken after I year of storage under dark conditions.

Abbreviations: h, hours; UV/Vis, ultraviolet-visible spectroscopy; HPC, hydroxypropylcellulose; NP, nanoparticle; SEM, scanning electron microscopy.

\section{Antimicrobial activity of Ag NPs}

The Ag NPs showed significant antimicrobial activity against the most commonly tested strains of bacteria and fungi (ie, S. epidermidis, S. aureus, P. aeruginosa, B. subtilis, E. coli, Actinomycetes, and A. niger). The zones of inhibition (radial diameter) for the aforementioned strains were found to be $26 \mathrm{~nm}, 29 \mathrm{~nm}, 33 \mathrm{~nm}, 33 \mathrm{~nm}, 20 \mathrm{~nm}, 22 \mathrm{~nm}$, and $21 \mathrm{~mm}$, respectively, using an $\mathrm{Ag}$ NP solution with a $50 \mathrm{mmol}$ concentration. Similarly, $75 \mathrm{mmol}$ and $100 \mathrm{mmol}$ solutions of Ag NPs were tested against the aforementioned strains, and the results are cumulatively shown in Figure 9. In simultaneously performed experiments for controls (HPC solution and sterile distilled water-filled cavities), there was no antimicrobial activity, whereas $\mathrm{AgNO}_{3}$ solution $(0.01 \mathrm{M})$ was found to be active against the mentioned strains. Antimicrobial assay was carried out in triplicate and the mean values have been reported. As a typical example, the antimicrobial activity of $\operatorname{Ag~NPs~}(50 \mathrm{mmol})$ against $P$. aeruginosa is shown in Figure 9, clearly indicating the zone of inhibition. However, polymer HPC and distilled water did not show any activity. The Ag NPs prepared by said novel and green method are potentially active against a variety of microbes; therefore, such pristine Ag NPs can be used as effective therapeutic tools.

\section{Conclusion}

The present study reports a green and low-cost method for the synthesis of Ag NPs based on HPC. The role of HPC in the formation and stabilization of Ag NPs has been 

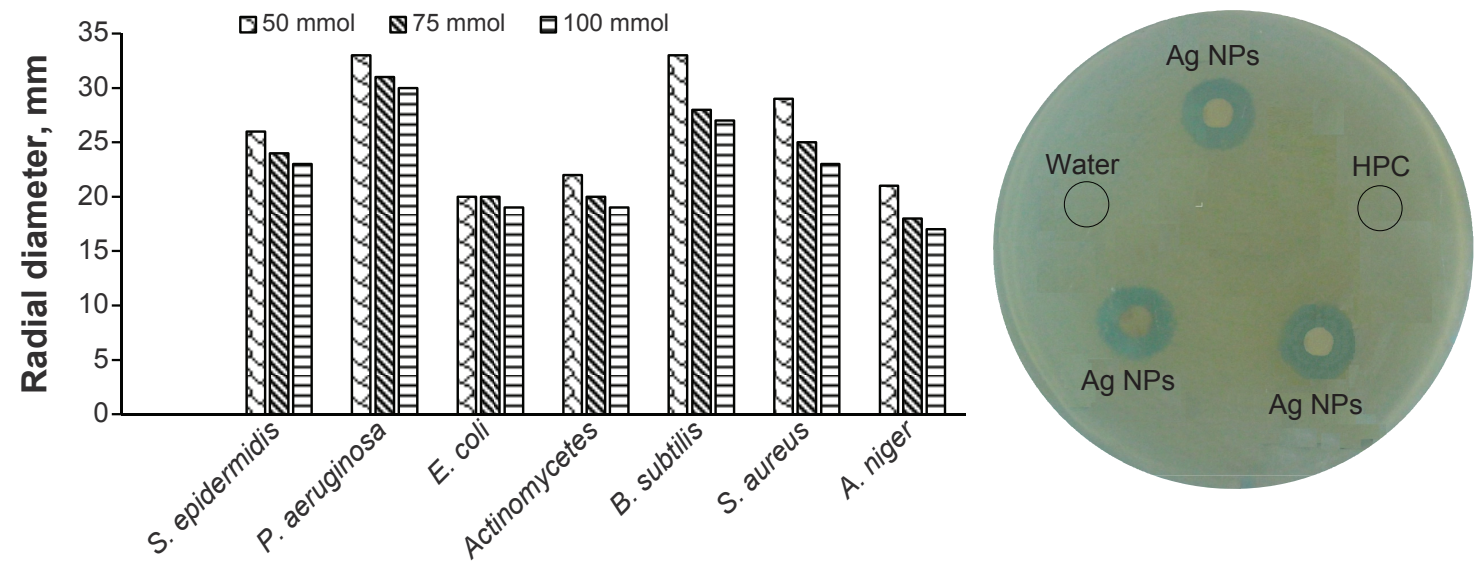

\section{Microbial strains}

Figure 9 Inhibitory zone of Ag NPs $(50 \mathrm{mmol}, 75 \mathrm{mmol}$, and $100 \mathrm{mmol})$ versus different bacterial and fungal strains.

Abbreviations: S. epidermidis, Staphylococcus epidermidis; P. aeruginosa, Pseudomonas aeruginosa; E. coli, Escherichia coli; B. subtilis, Bacillus subtilis; S. aureus, Staphylococcus aureus; A. niger, Aspergillus niger; NPs, nanoparticles; HPC, hydroxypropylcellulose.

investigated. $\mathrm{AgNO}_{3}$ solutions were reduced to $\mathrm{Ag} \mathrm{NPs}$ through a nontoxic and green reducing agent (ie, HPC). The stability of the Ag NPs after 1 year of storage has been achieved using HPC. The green synthesis of Ag NPs can be exploited as bioactive materials in biopolymer composites. The Ag NP-biopolymer composites have hybrid properties of both polymer and Ag NPs. Such hybrid materials could find potential applications in catalysis, nanobiotechnology, and therapeutics.

\section{Disclosure}

The authors report no conflicts of interest in this work.

\section{References}

1. Sun YP, Riggs JE, Rollins HW, Guduru R. Strong optical limiting of silver-containing nanocrystalline particles in stable suspensions. J Phys Chem B. 1999;103(1):77-82.

2. Kamat PV. Photophysical, photochemical and photocatalytic aspects of metal nanoparticles. $J$ Phys Chem B. 2002;106:7729-7744.

3. Oliveira MM, Ugarte D, Zanchet D, Zarbin AJ. Influence of synthetic parameters on the size, structure, and stability of dodecanethiolstabilized silver nanoparticles. J Colloid Interface Sci. 2005;292(2): 429-435.

4. Shipway AN, Katz E, Willner I. Nanoparticle arrays on surfaces for electronic, optical, and sensor applications. Chemphyschem. 2000;1(1): $18-52$

5. Hayward RC, Saville DA, Aksay IA. Electrophoretic assembly of colloidal crystals with optically tunable micropatterns. Nature. 2000; 404(6773):56-59.

6. Mfouo-Tynga I, El-Hussein A, Abdel-Harith M, Abrahamse H. Photodynamic ability of silver nanoparticles in inducing cytotoxic effects in breast and lung cancer cell lines. Int J Nanomedicine. 2014;9(1):3771-3780.

7. Zhao Q, Duan R, Yuan J, Quan Y, Yang H, Xi M. A reusable localized surface plasmon resonance biosensor for quantitative detection of serum squamous cell carcinoma antigen in cervical cancer patients based on silver nanoparticles array. Int J Nanomedicine. 2014;9:1097-1104.
8. Khan M, Khan ST, Khan M, et al. Antibacterial properties of silver nanoparticles synthesized using Pulicaria glutinosa plant extract as a green bioreductant. Int J Nanomedicine. 2014;9:3551-3565.

9. Seo Y, Hwang J, Kim J, Jeong Y, Hwang MP, Choi J. Antibacterial activity and cytotoxicity of multi-walled carbon nanotubes decorated with silver nanoparticles. Int J Nanomedicine. 2014;9:4621-4629.

10. Zheng J, Ding Y, Tian B, Wang ZL, Zhuang X. Luminescent and Raman active silver nanoparticles with polycrystalline structure. $J \mathrm{Am} C \mathrm{Chem}$ Soc. 2008;130(32):10472-10473.

11. Zhang Y, Zhang K, Ma H. Electrochemical DNA biosensor based on silver nanoparticles/poly(3-(3-pyridyl) acrylic acid)/carbon nanotubes modified electrode. Anal Biochem. 2009;387(1):13-19.

12. Eustis S, el-Sayed MA. Why gold nanoparticles are more precious than pretty gold: noble metal surface plasmon resonance and its enhancement of the radiative and nonradiative properties of nanocrystals of different shapes. Chem Soc Rev. 2006;35(3):209-217.

13. Dosescu D, Nistor CL, Purcar C, et al. Formation and dissolution of silver nanoparticles. Journal of Optoelectronics and Advanced Materials. 2009; $1: 44-48$

14. Tsuji T, Thang DH, Okazaki Y, Nakanishi M, Tsuboi Y, Tsuji M. Preparation of silver nanoparticles by laser ablation in polyvinylpyrrolidone solutions. Appl Surf Sci. 2008;254(16):5224-5230.

15. Shameli K, Ahmad MB, Yunus WM, Ibrahim NA, Gharayebi Y, Sedaghat S. Synthesis of silver/montmorillonite nanocomposites using $\gamma$-irradiation. Int J Nanomedicine. 2010;5:1067-1077.

16. Oves M, Khan MS, Zaidi A, et al. Antibacterial and cytotoxic efficacy of extracellular silver nanoparticles biofabricated from chromium reducing novel OS4 strain of Stenotrophomonas maltophilia. PLoS One. 2013;8(3):e59140

17. Shameli K, Ahmad MB, Yunus WM, et al. Green synthesis of silver/ montmorillonite/chitosan bionanocomposites using the UV irradiation method and evaluation of antibacterial activity. Int $J$ Nanomedicine. 2010;5:875-887.

18. Pastroiza-Santos I, Liz-Marzan LM. Formation of PVP-protected metal nanoparticles in DMF. Langmuir. 2002;18(7):2888-2894.

19. Chen M, Wang LY, Han JT, Zhang JY, Li ZY, Qian DJ. Preparation and study of polyacryamide-stabilized silver nanoparticles through a one-pot process. J Phys Chem B. 2006;110(23):11224-11231.

20. Kuo PL, Chen WF. Formation of silver nanoparticles under structured amino groups in pseudo-dendritic poly(allylamine) derivatives. $J$ Phys Chem B. 2003;107:11267-11272. 
21. Zou X, Bao H, Guo H, et al. Mercaptoethane sulfonate protected, water-soluble gold and silver nanoparticles: syntheses, characterization and their building multilayer films with polyaniline via ion-dipole interactions. J Colloid Interface Sci. 2006;295(2):401-408.

22. Popa M, Pradell T, Crespo D, Calderón-Moreno JM. Stable silver colloidal dispersions using short-chain polyethylene glycol. Colloids Surf A Physicochem Eng Asp. 2007;303(3):184-190.

23. Vimala K, Samba Sivudu K, Murali Mohan Y, Sreedhar B, Mohana Raju K. Controlled silver nanoparticles synthesis in semi-hydrogel networks of poly(acrylamide) and carbohydrates: a rational methodology for antibacterial application. Carbohydr Polym. 2009; 75(3):463-471.

24. Murphy CJ. Sustainability as an emerging design criterion in nanoparticle synthesis and applications. J Mater Chem. 2008;18:2173-2176.

25. Prozorova GF, Pozdnyakov AS, Kuznetsova NP, et al. Green synthesis of water-soluble nontoxic polymeric nanocomposites containing silver nanoparticles. Int J Nanomedicine. 2014;9:1883-1889.

26. Muzzarelli RAA. Genipin-crosslinked chitosan hydrogels as biomedical and pharmaceutical aids. Carbohydr Polym. 2009;77(1):1-9.
27. El-Sheikh MA, El-Rafie SM, Abdel-Halim ES, El-Rafie MH. Green synthesis of hydroxyethyl cellulose-stabilized silver nanoparticles. Journal of Polymers. 2013;650837.

28. Abdel-Halim ES, Al-Deyab SS. Utilization of hydroxypropyl cellulose for green and efficient synthesis of silver nanoparticles. Carbohydr Polym. 2011;86(4):1615-1622.

29. Hebeish AA, El-RafieMH, Abdel-MohdyFA, Abdel-HalimES, EmamHE. Carboxymethyl cellulose for green synthesis and stabilization of silver nanoparticles. Carbohydr Polym. 2010;82(3):933-941.

30. Angelova T, Rangelova N, Yuryev R, Georgieva N, Müller R. Antibacterial activity of $\mathrm{SiO}$ /hydroxypropyl cellulose hybrid materials containing silver nanoparticles. Mater Sci Eng C Mater Biol Appl. 2012; 32(5):1241-1246.

31. Mock JJ, Barbic M, Smith DR, Schultz DA, Schultz S. Shape effects in plasmon resonance of individual colloidal silver nanoparticles. J Chem Phys. 2002;116:6755-6759.
International Journal of Nanomedicine

\section{Publish your work in this journal}

The International Journal of Nanomedicine is an international, peerreviewed journal focusing on the application of nanotechnology in diagnostics, therapeutics, and drug delivery systems throughout the biomedical field. This journal is indexed on PubMed Central, MedLine, CAS, SciSearch $®$, Current Contents ${ }^{\circledR} /$ Clinical Medicine,

\section{Dovepress}

Journal Citation Reports/Science Edition, EMBase, Scopus and the Elsevier Bibliographic databases. The manuscript management system is completely online and includes a very quick and fair peer-review system, which is all easy to use. Visit http://www.dovepress.com/ testimonials.php to read real quotes from published authors. 